[white paper]

Diamond Open Access

\title{
O que é matéria escura? Ela realmente existe?
}

\author{
Colaboração Física Aberta ${ }^{1}$ \\ 25 de Julho de 2021
}

\begin{abstract}
Resumo
Este é um artigo de divulgação científica em que discutimos, resumidamente, a partir de [1], o que é matéria escura e se ela realmente existe.
\end{abstract}

palavras-chave: cosmologia, matéria escura, gravitação quântica, galáxia

A versão mais atualizada deste artigo está disponível em https://osf.io/thm4p/download https://zenodo.org/record/5136459

\section{Preâmbulo}

1. Este artigo foi elaborado a partir de [1], seguindo as diretrizes da licença Creative Commons [2].

2. Sobre a história da matéria escura, veja [3].

3. A referência [1], em português, pode ser lida em https://bit.1y/3BD4CTJ.

${ }^{1}$ Todos os autores com suas afiliações aparecem no final deste artigo. 


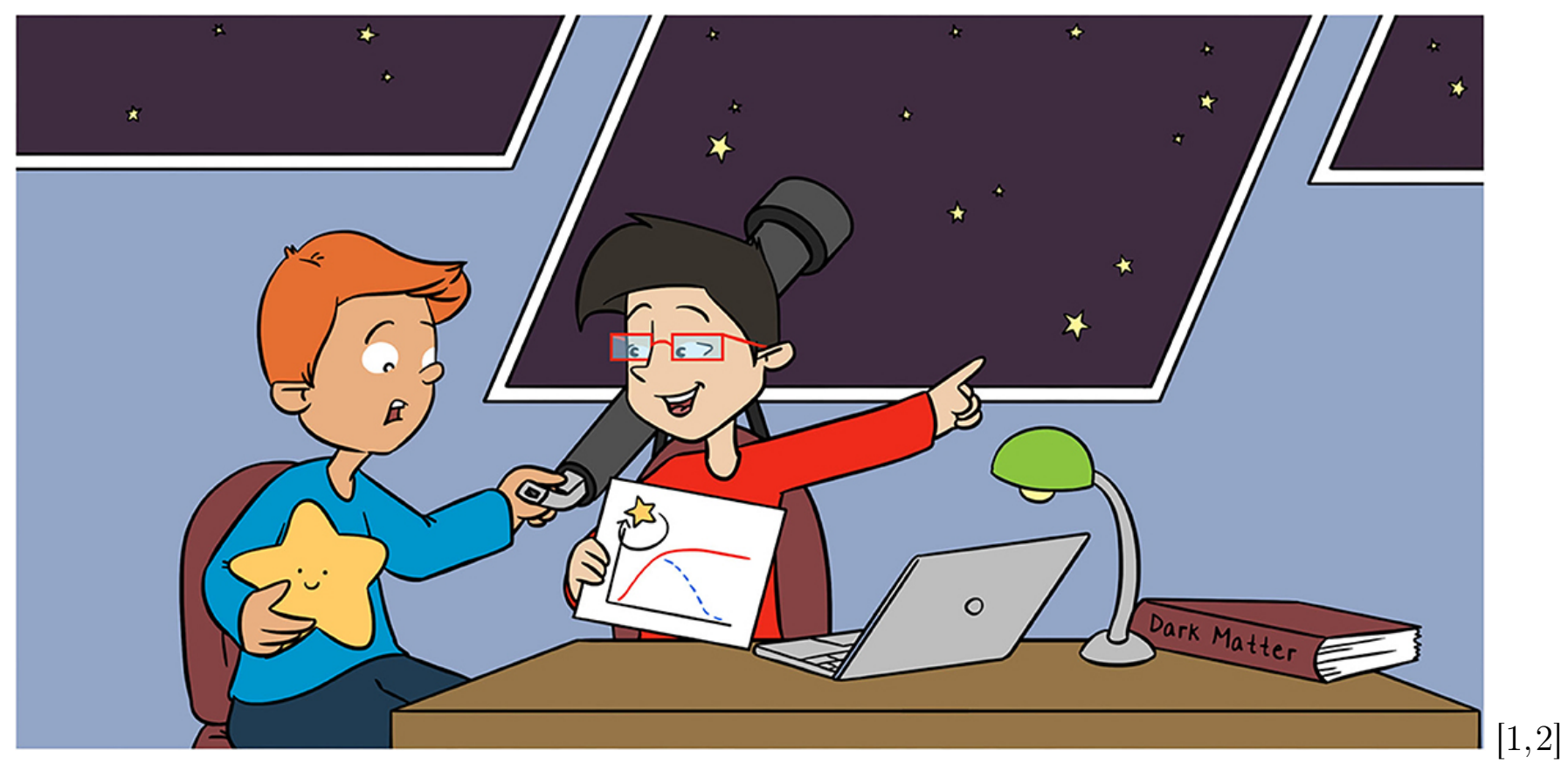

\section{Desafios}

4. O universo é inimaginavelmente maior do que pensamos e, por isso, ainda há muita coisa que não sabemos sobre seu funcionamento.

5. Compreender a existência e a composição de uma substância misteriosa chamada matéria escura é um dos principais desafios que os cientistas enfrentam hoje.

6. Existem muitas teorias sobre o que poderia ser a matéria escura, mas ainda precisamos entender sua verdadeira natureza.

7. Como podemos saber se realmente existe a matéria escura?

8. O maior desafio para estudar a matéria escura é que não podemos vê-la diretamente; conseguimos apenas observar seu efeito indireto.

9. Neste artigo, discutiremos como os cientistas usam a ciência e as observações de telescópios para prever a existência de matéria escura e por que os cientistas pensam que ela permeia nosso universo. 


\section{Glossário}

10. Espectro eletromagnético

A gama completa de frequências de luz, de ondas de rádio a raios gama e raios- $X$.

11. Comprimento de onda

Uma medida da luz, especificamente a distância entre os picos nas ondas de luz. Os comprimentos de onda da luz são medidos em nanômetros (nm) e variam de cerca de $400 \mathrm{~nm}$ (ultravioleta) a $700 \mathrm{~nm}$ (infravermelho), com a luz visivel no meio.

12. Matéria

Qualquer coisa que tenha massa.

13. Matéria escura

Matéria que não emite luz e, portanto, não pode ser vista com telescópios.

\section{Astrofísico}

Cientista que estuda objetos astronômicos, até o tamanho de todo o universo.

15. Velocidade

A distância percorrida entre dois pontos por unidade de tempo. Por exemplo, um carro se movendo a $60 \mathrm{~km} / \mathrm{h}$ viaja $60 \mathrm{~km}$ do ponto $A$ ao ponto $B$ em 1 h.

16. Lente gravitacional

A luz produzida por galáxias distantes se curva e se distorce à medida que interage com o campo gravitacional de grandes quantidades de massa, como aglomerados de galáxias. 
17. Neutrinos

Partículas minúsculas, menores que átomos, que não possuem carga elétrica. Os neutrinos podem ser um dos componentes da matéria escura.

\section{O que é matéria escura?}

18. No universo, os corpos celestes que brilham podem ser planetas em nosso sistema solar, estrelas em nossa galáxia e galáxias inteiras que estão bem distantes.

19. Esses objetos constituem toda a matéria emissora de luz do universo.

20. Com a ajuda de diferentes tipos de telescópios, podemos observar esses corpos celestes por meio da luz que eles irradiam.

21. Todos os telescópios funcionam detectando luz no espectro eletromagnético, da luz visível aos raios X, emitidos por esses corpos celestes.

22. Os cientistas usam os vários comprimentos de onda da luz detectadas para determinar as principais informações sobre os corpos celestes do nosso universo, como distância, idade, tamanho e forma.

23. Eles podem até usar algumas dessas informações para compreender as leis do universo.

24. No entanto, há matéria no universo que não emite luz em nenhuma parte do espectro eletromagnético, o que significa que não podemos observá-la com nossos telescópios.

25. Esta propriedade singular torna impossível observar esses tipos de matéria diretamente, então os cientistas chamam de matéria escura. 


\section{A matéria escura existe?}

26. Alguns cientistas, especificamente os astrofísicos, passam muito tempo produzindo teorias sobre o que poderia ser a matéria escura.

27. Os cientistas sabem que a matéria escura não emite luz de nenhuma parte do espectro eletromagnético, mas observou-se que a matéria escura é influenciada pela gravidade.

28. Os astrofísicos ainda não têm certeza do que é, exatamente, a matéria escura.

29. No entanto, eles sabem o que a matéria escura não é, observando como ela se comporta em comparação com outros materiais.

30. Sabemos que a matéria escura constitui, aproximadamente, $80 \%$ da massa total das galáxias [4].

31. Isso significa que há quatro vezes mais matéria escura em comparação com a matéria normal!

32. Se a matéria escura é tão difícil de observar, por que os cientistas acreditam que ela realmente existe?

33. A evidência para apoiar a existência de matéria escura é extensa e exploraremos os dois principais exemplos nas seções seguintes.

34. No entanto, pode ser que não exista matéria escura.

35. Outra alternativa seria a de que nossas teorias que descrevem a gravidade estão incompletas, e uma boa revisão delas será necessária para que a gente consiga explicar os fenômenos gravitacionais observados nas galáxias. 


\section{O movimento das estrelas nas galáxias}

36. O primeiro tipo de evidência que apóia a existência de matéria escura tem a ver com a maneira como essa suposta matéria escura afeta o movimento dos corpos celestes.

37. Em nosso sistema solar, quase toda a massa está no Sol.

38. Os planetas mais internos, como Mercúrio e Vênus, orbitam o Sol mais rápido.

39. À medida que a distância do Sol aumenta, a velocidade com que os planetas se movem diminui.

40. Isso ocorre porque há menos atração gravitacional do Sol em planetas mais distantes e, para evitar uma espiral para dentro ou para longe do Sol, eles devem se mover mais devagar.

41. Podemos aplicar uma analogia semelhante às galáxias.

42. Se assumirmos que a parte brilhante de uma galáxia mostra onde está a maior parte da massa, então a maior parte da massa está perto do centro, e na borda escura de uma galáxia não deve haver muita massa.

43. Portanto, objetos orbitando longe do centro da galáxia devem se mover mais devagar do que objetos mais próximos do centro, assim como os planetas em nosso sistema solar.

44. Para testar essa hipótese, os cientistas registraram a luz que chega de uma galáxia espiral distante (nossa galáxia, a Via Láctea, também é considerada uma galáxia espiral) e traçaram as velocidades das estrelas versus suas distâncias do centro da galáxia.

45. Os cientistas descobriram que as estrelas não estavam se comportando da maneira esperada.

46. Eles descobriram que as estrelas mais distantes do centro estavam se movendo muito mais rápido do que o previsto (Fig. 1). 
47. Uma maneira disso ser possivel é se houver mais massa nas partes externas das galáxias do que podemos observar.

48. O fato de não sermos capazes de ver essa massa, por ela não estar emitindo luz, sugere a presença de matéria escura, como uma possivel explicação.

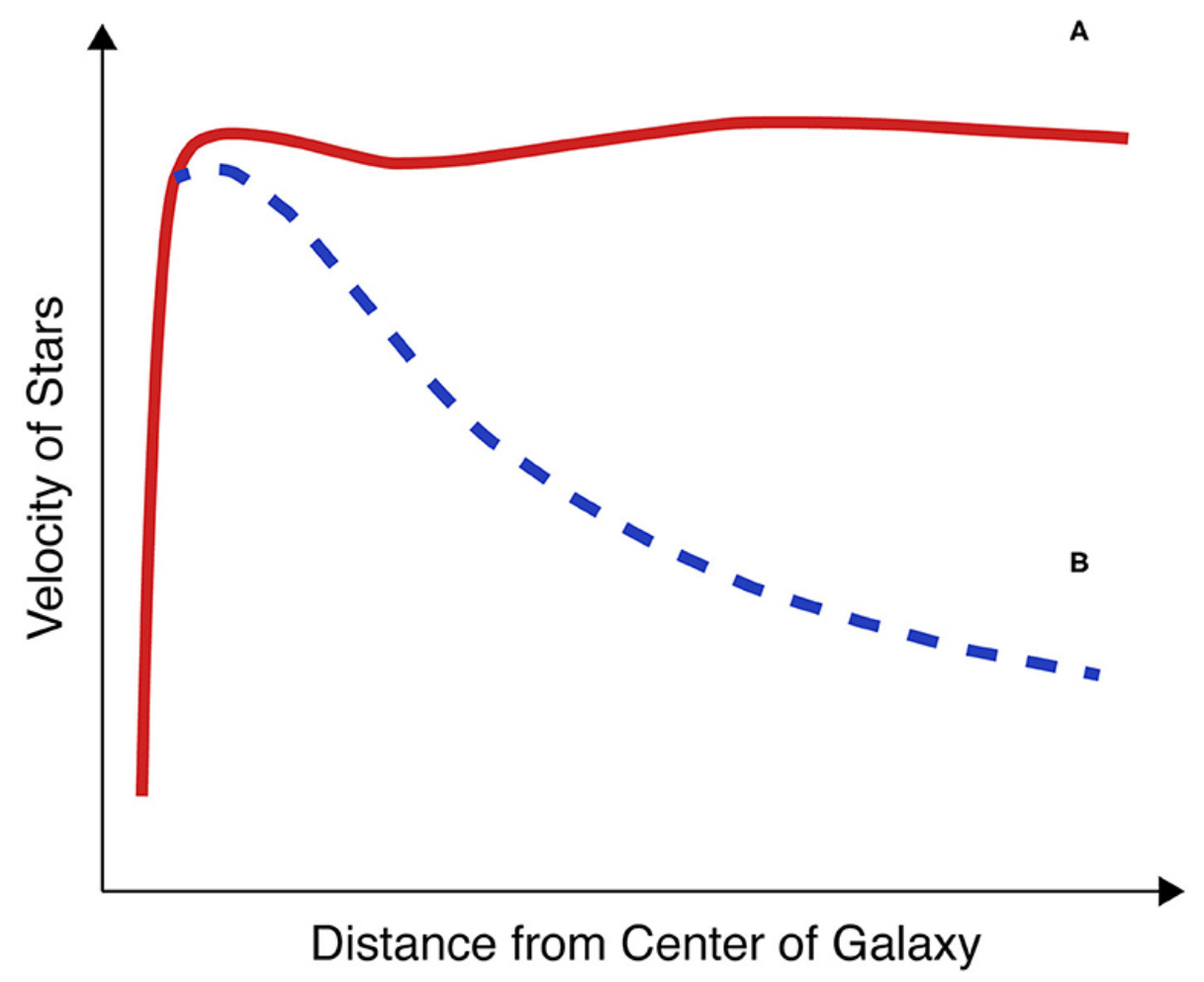

Figura 1: Relação entre a velocidade das estrelas e sua distância do centro da galáxia. A velocidade real observada das estrelas (A) que estão longe do centro da galáxia é maior do que o previsto pelos cientistas (B). (Modificado da imagem original por PhilHibbs e licenciado sob CC BY-SA $3,0)[2,5]$.

49. Os dados da Fig. 1 indicam que pode haver uma grande quantidade de massa presente na parte externa da galáxia que não podemos ver porque ela não emite luz, sugerindo a presença de matéria escura. 


\section{A curvatura da luz}

50. A terceira evidência que apoia a existência de matéria escura vem de um estudo do Bullet Cluster, que é o nome dado a duas galáxias que colidiram recentemente.

51. Os astrônomos descobriram uma maneira de descobrir a massa de um objeto celeste, como uma galáxia, usando uma técnica conhecida como lente gravitacional [6].

52. A lente gravitacional é fundamentada no fato de que a massa de um objeto influencia a densidade do espaço ao seu redor.

53. Quando a luz viaja por um espaço denso, ela se curva.

54. Quando a luz passa perto de um objeto no espaço, ela viaja na superfície curva, que curva as ondas de luz.

55. Quanto maior a massa do objeto, mais a luz se curva.

56. Com a ajuda dessa teoria, podemos determinar a massa de um objeto celeste observando o quanto a luz de uma estrela logo atrás dele se curva.

57. Usando lentes gravitacionais, os cientistas determinaram a massa total do Bullet Cluster, incluindo a matéria escura [7].

58. A Fig. 2 mostra que a maior parte da massa do aglomerado Bullet não está localizada onde a fonte das emissões de raios-X se originam, o que significa que não é da matéria que podemos ver.

59. Consequentemente, essas galáxias são compostas por muito mais matéria escura do que de matéria regular.

60. Nesse modelo, está implícita a premissa de que a matéria escura gera um campo gravitacional, tal como a matéria ordinária. 


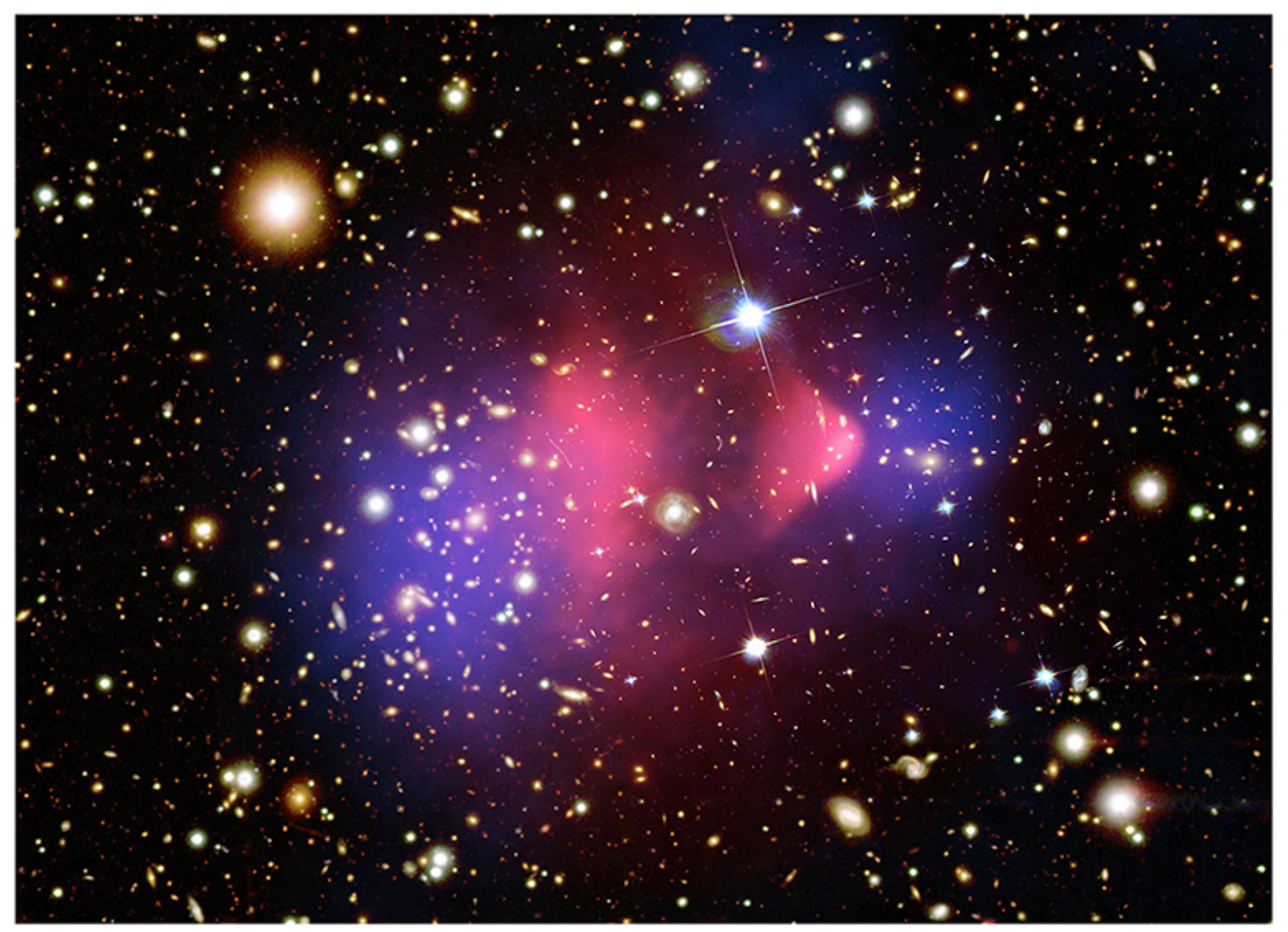

Figura 2: Uma imagem do Bullet Cluster produzida pelo Telescópio Espacial Hubble da NASA e pelo Observatório Chandra de Raios-X. O Bullet Cluster é uma combinação de duas galáxias que uma vez colidiram. As áreas azuis representam a maior parte da massa das galáxias, que é composta por matéria escura, e as áreas rosa são matéria ordinária. Podemos detectar a matéria escura através de lentes gravitacionais, que detectam mudanças na luz produzidas por objetos celestes distantes [7]. Os pontos brilhantes fora das áreas coloridas são estrelas e galáxias que não fazem parte do Bullet Cluster. Os créditos da imagem estão em [1]. 


\section{O que poderia ser a matéria escura?}

61. Afinal, do que é feita a matéria escura, caso ela exista?

62. Os cientistas já descobriram um tipo de partícula que supostamente compõe a matéria escura, os neutrinos.

63. Se a matéria escura realmente existir, os neutrinos seriam um dos componentes da matéria escura.

64. Neutrinos são partículas que não emitem luz, assim como a matéria escura.

65. No entanto, os neutrinos podem representar apenas uma fração da quantidade total de matéria escura, porque são muito claros e, quando foram criados no início do universo, estavam se movendo muito rápido.

66. Portanto, outras partículas ainda a serem descobertas devem estar envolvidas.

67. Dois dos candidatos mais promissores são as partículas chamadas WIMPS e áxions.

68. Nenhuma dessas partículas foram observadas ainda; muitos experimentos ao redor do mundo estão procurando por elas.

\section{Considerações Finais}

69. A matéria escura representa cerca de $63 \%$ de toda a matéria do universo (Fig. 3).

70. Nossa capacidade de compreender a matéria escura nos ajudará a aprender mais sobre o universo, incluindo detalhes sobre sua origem e formação. 
71. Muitos experimentos estão sendo realizados em todo o mundo, incluindo experimentos do Large Hadron Collider na Suíça, para determinar a natureza das partículas minúsculas que podem nos dizer mais sobre as condições em que a matéria escura é formada.

72. Mais trabalhos precisam ser feitos, há muito o que se descobrir na astrofísica e na física de partículas!

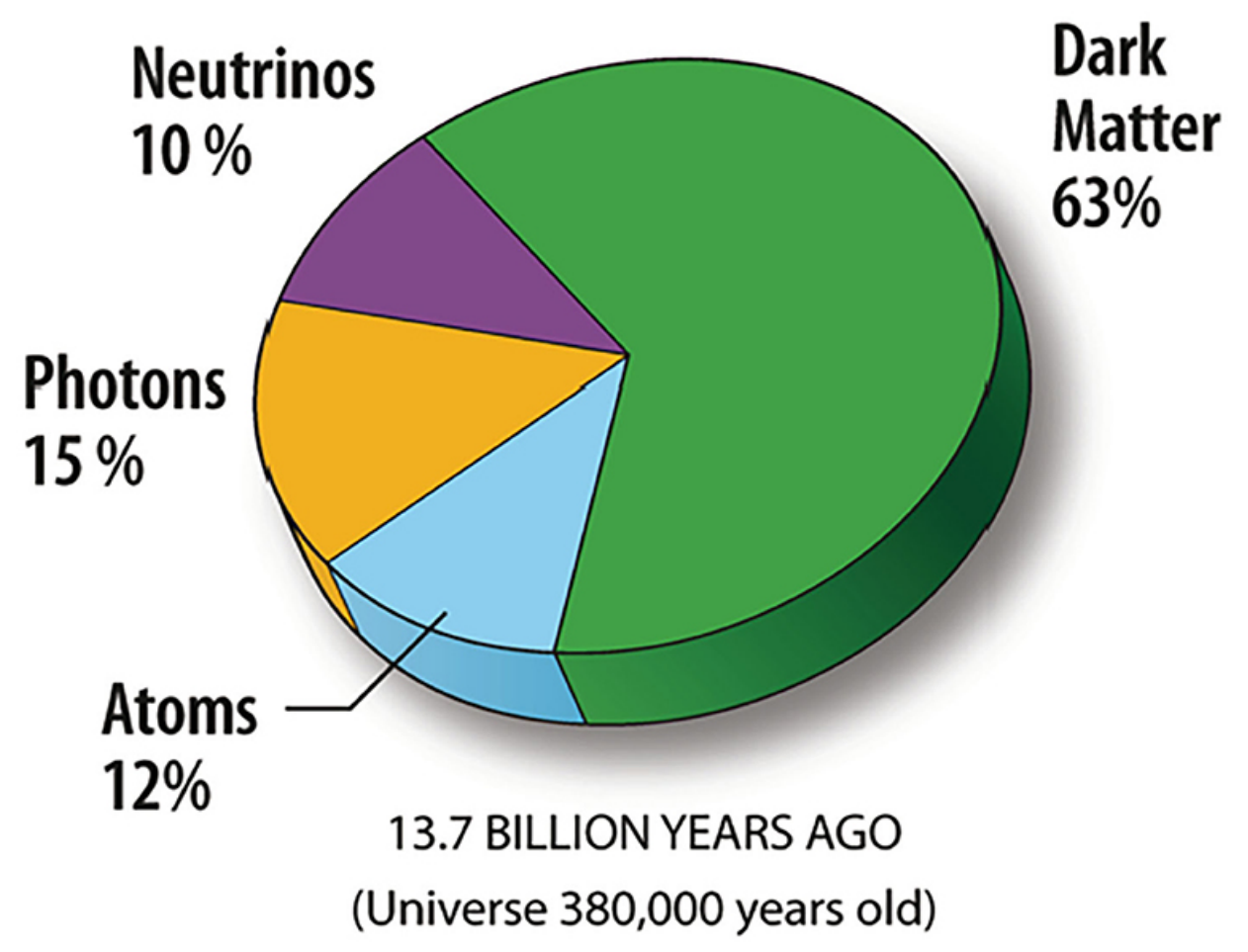

Figura 3: O círculo inteiro representa toda a matéria que estava presente 380 mil anos após o nascimento do universo. Você pode ver que a quantidade de matéria escura é muito maior do que todos os outros tipos de matéria. A quantidade de matéria escura permanece semelhante atualmente [4], e a massa do universo não mudou muito desde a era mostrada na figura. Este gráfico, criado pela $N A S A / W M A P$ Science Team, é de domínio público. 


\section{Ciência Aberta}

O arquivo latex para este artigo, juntamente com outros arquivos suplementares, estão disponíveis em [8]. Seja coautor(a) deste artigo, envie sua contribuição para mplobo@uft.edu.br.

\section{Consentimento}

O autor concorda com [9].

\section{Como citar este artigo?}

https://doi.org/10.31219/osf .io/thm4p

https://zenodo.org/record/5136459

\section{Referências}

[1] Bhathe V, Brennan C, Ellis S, Moynes E, Graham K and Landsman S (2021) "How Do Scientists Know Dark Matter Exists?." Front. Young Minds. 9:576034. https://doi .org/10.3389/frym.2021.576034

[2] CC. Creative Commons. Attribution 4.0 International (CC BY 4.0) https://creativecommons .org/licenses/by/4.0

[3] Bertone, G. and Hooper, D. 2018. "History of dark matter." Rev. Mod. Phys. 90:045002.

https://doi.org/10.1103/revmodphys.90.045002

[4] Ibarra, A. 2015. "Dark matter theory." Nucl. Part Phys. Proc. 267-269:323-31.

https://doi.org/10.1016/j.nuclphysbps.2015.10.126

[5] Garner, R. 2015. About the Hubble Space Telescope. https://www.nasa.gov/mission_pages/hubble/story 
[6] Wambsganss, J. 1998. "Gravitational lensing in astronomy." Living Rev. Relativ. 1:12. https://doi.org/10.12942//rr-1998-12

[7] Clowe, D., Bradač, M., Gonzalex, A. H., Markevitch, M., Randall, S. W., and Jones, C., et al 2006. "A direct empirical proof of the existence of dark matter." Astrophys. J. Lett. 648:L109-13.

https://doi.org/10.1086/508162

[8] Lobo, Matheus P. "Open Journal of Mathematics and Physics (OJMP)." OSF, 21 Apr. 2020.

https://doi.org/10.17605/osf .io/6hzyp

[9] Lobo, Matheus P. "Simple Guidelines for Authors: Open Journal of Mathematics and Physics." OSF Preprints, 15 Nov. 2019.

https://doi.org/10.31219/osf .io/fk836

\section{Colaboração Física Aberta}

Matheus Pereira Lobo (autor principal, mplobo@uft.edu.br) $)^{1,2}$ https://orcid.org/0000-0003-4554-1372

${ }^{1}$ Universidade Federal do Tocantins (Brasil)

${ }^{2}$ Universidade Aberta (UAb, Portugal) 